\title{
BMJ Open Combination of obstructive sleep apnoea and insomnia treated by continuous positive airway pressure with the SensAwake pressure relief technology to assist sleep: a randomised cross-over trial protocol
}

\author{
Jean Louis Pepin, ${ }^{1}$ Frédéric Gagnadoux, ${ }^{2}$ Alison Foote, ${ }^{1}$ Rachel Vicars, ${ }^{3}$ \\ Bhavi Ogra, ${ }^{3}$ Véronique Viot-Blanc, ${ }^{4}$ Meriem Benmerad, ${ }^{1}$ Marie-Pia D'Ortho, ${ }^{5}$
} Renaud Tamisier ${ }^{1}$

To cite: Pepin JL, Gagnadoux F, Foote A, et al. Combination of obstructive sleep apnoea and insomnia treated by continuous positive airway pressure with the SensAwake pressure relief technology to assist sleep: a randomised crossover trial protocol. BMJ Open 2017;7:e015836. doi:10.1136/ bmjopen-2017-015836

- Prepublication history and additional material for this paper are available online. To view these files, please visit the journal online (http://dx.doi. org/10.1136/bmjopen-2017015836).

Received 3 January 2017 Revised 28 July 2017 Accepted 29 August 2017

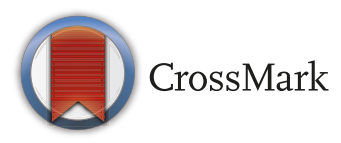

For numbered affiliations see end of article.

Correspondence to

Dr Alison Foote;

AFoote@chu-grenoble.fr

\section{ABSTRACT}

Introduction Obstructive sleep apnoea (OSA) is a common sleep breathing disorder affecting up to $17 \%$ of the middle-aged population. Continuous positive airway pressure (CPAP) is the primary treatment for patients with OSA, but acceptance and adherence to therapy is suboptimal in specific subgroups particularly those with insomnia or poor sleep quality $(40 \%-80 \%$ of patients with OSA). Pressure intolerance, particularly during periods of wakefulness, inhibiting sleep onset or return to sleep, is one reason for poor CPAP adherence. AutoCPAPs continually monitor airflow changes and only increase the pressure when the upper airway requires it. Reducing the pressure during wakefulness-sleep transition and wakefulness-after-sleep-onset (WASO) may improve therapy comfort and potentially adherence without compromising therapy efficacy. We hypothesise that SensAwake, a pressure relief function that reduces CPAP pressure on the transition from sleep to wakefulness and on WASO, may improve objective sleep quality.

Methods and analysis This is a multicentre, randomised double-blind crossover clinical trial on patients with both OSA and insomnia. Insomnia is defined as Insomnia Severity Index $>15$ at screening. Baseline data, including actigraphy, are collected for 1 week before randomisation (1:1) to either conventional AutoCPAP or AutoCPAP with SensAwake for 4 weeks. After an evaluation visit, patients are switched to the other treatment arm for a further 4 weeks. Allowing for $20 \%$ dropout, 48 patients are required. If applicable, repeated measures analysis of variance will be used to assess differences in WASO measured by actigraphy (primary outcome), other actigraphy measures, AutoCPAP compliance, subjective questionnaire scores (Epworth Sleepiness Scale, Pittsburgh Sleep Quality Index, Short-Form 12 Health Survey) and 24 hours blood pressure (secondary outcomes).

Ethics and dissemination The protocol was approved by the regional Ethics Committee (CPP Sud-Est-V, IRB $\mathrm{N}^{\circ}$ 6705) on 9 December 2015, is registered on ClincalTrials.gov (NCT02721329) and started in June 2016
Strengths and limitations of this study

- This multicentre randomised crossover trial could potentially lead to an alternative to conventional Autocontinuous positive airway pressure (CPAP) therapy in a well-defined common obstructive sleep apnoea (OSA) having a high rate of CPAP noncompliance.

- While the sample size is just sufficient to analyse objective sleep indices (primary outcome) in a crossover design, it is too small to inform on 24 hours blood pressure and patient-centred outcomes (secondary outcomes).

- The wrist actimeter used has not been previously validated for the measurement of wake-after-sleeponset in this specific patient phenotype associating OSA and insomnia.

- Only one night of blood pressure monitoring is done in each arm of the study. Longer Ambulatory Blood Pressure Monitoring (ABPM) would be desirable, but wearing yet another device may bias the study.

- Despite having several study sites the sample size is rather small, one limitation being the difficulties in recruitment of willing patients naïve to CPAP therapy; restricting the relevance of any secondary analyses such as those on blood pressure.

with expected publication of primary outcome results in 2018.

Trial registration number NCT0272132; Pre-results.

\section{INTRODUCTION}

Obstructive sleep apnoea (OSA) is a common sleep breathing disorder affecting up to $17 \%$ of the middle-aged population ${ }^{12}$ and is characterised by periodic collapse of the upper airway during sleep. Continuous positive 
airway pressure (CPAP) is the primary treatment for patients with OSA. ${ }^{34}$ Despite the effectiveness of CPAP in abolishing upper airway obstruction, acceptance of and adherence to therapy is often suboptimal particularly in specific phenotypes, including the combination of OSA and insomnia ${ }^{56}$ or insomnia symptoms. ${ }^{7}$

Pressure intolerance is one possible reason for this lack of adherence. Conventional CPAP generally delivers higher pressure than necessary for much of the night as the needed CPAP pressure is selected based either on one night's titration (in a sleep laboratory) or during several nights at home and pressure requirements can vary considerably with sleeping posture, sleep stage and environmental influences such as alcohol and sedative use. ${ }^{89}$ AutoCPAPs address this problem by continually monitoring airflow changes and only increasing the pressure when the upper airway requires it. Research suggests that AutoCPAP generally delivers an overall lower mean treatment pressure than conventional CPAP. ${ }^{10-16}$ Despite this, there is limited evidence to suggest that AutoCPAP therapy can considerably improve CPAP adherence and acceptance in an unselected population, but this might be different in patients with OSA with concurrent insomnia. ${ }^{10} 1417-23$

Conceptually, a patient's awareness of pressure occurs only during wakefulness. Thus, reducing the pressure during wakefulness may improve therapy comfort and potentially adherence without compromising therapy efficacy. SensAwake (Fisher \& Paykel Healthcare, Auckland, New Zealand) is a pressure relief technology that accurately detects irregularity in the flow signal indicative of the transition from sleep to wake. ${ }^{24}$ When the transition from sleep to wake is detected, the device promptly reduces the pressure to help facilitate a return to sleep. CPAP/AutoCPAP with SensAwake has been used in the general OSA population and has been shown to provide the same treatment efficacy at a lower overall pressure as CPAP/AutoCPAP without SensAwake 25 and patients have judged it to be more comfortable and preferred it to CPAP without SensAwake. ${ }^{27}$

The prevalence of insomnia symptoms in patients with OSA is estimated to be $40 \%-80 \%,{ }^{72-32}$ and existence of insomnia has been shown to negatively affect CPAP compliance in some studies. ${ }^{334}$ It is proposed that insomnia patients are preoccupied with external factors that may be perceived as a threat to sleep, which results in a higher wake-after-sleep-onset (WASO), the amount of time a person spends awake from when they first fall asleep to when they do not attempt to go back to sleep. Besides wakening it takes account of difficulty in getting back to sleep, which may be further exacerbated by the presence of CPAP. ${ }^{33}$ It is therefore hypothesised that the pressure relief that SensAwake provides during wakefulness may be of a greater benefit to patients with OSA and insomnia if it can facilitate the return to sleep. There is no known published data on the use of SensAwake in the OSA/insomnia population.

\section{Primary research objective}

The primary objective is to compare the at-home objective sleep quality (WASO) when using AutoCPAP with SensAwake versus AutoCPAP without SensAwake in patients with a diagnosis of OSA and insomnia.

\section{Secondary research objectives}

The secondary objectives are to compare AutoCPAP compliance, other measures of objective sleep quality (total sleep time (TST), sleep onset latency (SOL) and sleep efficiency (SE)), daytime sleepiness, subjective sleep quality, insomnia, quality of life and 24 hours blood pressure.

\section{METHODS AND ANALYSIS}

\section{Study design}

This is multicentre prospective 1:1 randomised, doubleblind, crossover trial.

\section{Patient entry and screening for insomnia}

Outpatients diagnosed with OSA by polysomnography at one of the participating tertiary hospital sleep centres (Grenoble, Angers and Bichat and Lariboisière hospitals in Paris, France) between November 2016 and October 2017, eligible for CPAP treatment under local requirements (Apnoea Hypoxia Index $>30$ with no more than $20 \%$ central respiratory events) are asked to answer an Insomnia Severity Index (ISI) questionnaire ${ }^{35}$ to screen for insomnia. If they meet the study inclusion/non-inclusion criteria (box), they are asked by the sleep physician for their written informed consent (available as a Supplementary file 1) and are enrolled into the study.

\section{Materials}

Nighttime actigraphy, the gold standard for measuring objective sleep quality in the home, is recorded using a wGT3X-BT wrist monitor from ActiGraph, (Pensacola, Florida, USA). This is a standard actigraphy device that uses an adapted version of the Cole-Kripke Algorithm. ${ }^{3637}$

The AutoCPAP device is the ICON+Auto from Fisher \& Paykel Healthcare. This has an integrated heated humidification system and is intended for use on adult patients for the treatment of OSA at home or in a sleep laboratory. The ICON+ treats OSA by delivering a continuous flow of air at a pressure prescribed by the physician to maintain the airway open. In AutoCPAP mode, the device auto-adjusts the therapeutic pressure between a set minimum and maximum in response to respiratory events (apnoea, hypopnoea and flow limitation). SensAwake responsive pressure relief technology is a comfort feature that is available in the ICON+Autodevice. It functions by detecting wakefulness using the flow signal and promptly reduces the pressure to a more comfortable level to allow the patient to return to sleep. The ICON+ recordsand reports industry standard metrics such as adherence, leaks and treatment efficacy data. 


\section{Box}

Inclusion criteria
Age $>18$ years.
Diagnosed with OSA and eligible for CPAP treatment under local
requirements (Apnoea Hypoxia Index $>30$ with no more than $20 \%$ of
central respiratory events).
Naïve to CPAP therapy, ie, have not been prescribed, or used CPAP
in the last 5 years
Insomnia Severity Index score $>15$
Fluent in spoken and written French
Exclusion criteria
Significant uncontrolled cardiac disease and/or left ventricular
ejection fraction $<45 \%$ and/or severe lung disease
Co-existing sleep disorder, such as predominant central sleep
apnoea, previous or current diagnosis of sleep phase delay
Pregnancy
Patient receiving cognitive behavioural therapy or other intervention
to treat insomnia. Subjects may be using hypnotics, but there shall
be no change in hypnotic use during the protocol or during the 4
weeks preceding enrolment into the study
Diagnosed with clinical depression and/or Hospital Anxiety and
Depression score $>11$ and/or currently using antidepressants and/
or anxiolytics within the last 6 months
Participating in another clinical trial for the duration of participation
in this study
Patient protected by the law, under guardianship or curatorship
Patient unwilling to give informed consent

The choice of CPAP mask is left to the patient, physician and/or home care provider and is the same as for usual care with CPAP.

Twenty-fourhours ambulatory blood pressure monitors are those normally used by the centres and are fitted and data collected by qualified clinical research assistants (CRA) blinded to the study arms during weeks 1, 5 and 9 of the study.

Answers to the validated French versions of the self-reported questionnaires: ISI, Epworth Sleepiness Scale (ESS) ${ }^{38}$ Pittsburgh Sleep Quality Index (PSQI) ${ }^{39}$ and Short-Form 12 Health Survey (SF-12) ${ }^{40}$ will be analysed as recommended by the authors of the questionnaires.

\section{Baseline data collection}

All patients wear a wrist actimeter for 1 week to record baseline sleep data. Participants are issued with and trained in the use of the actimeter and a sleep diary. The diary (from Fisher \& Paykel Healthcare) requires participants to record each morning what time they went to bed, what time they went to sleep, what time they woke up and what time they got up. During this week, blood pressure is monitored for one 24-hour period. Patients return to the centre for download of actigraphy data and randomisation. They complete the following questionnaires: ISI, ESS, PSQI and SF-12 supervised by a CRA.

\section{Randomisation}

Participants are block randomised via a secure electronic website to receive AutoCPAP either with or without SensAwake.

- SensAwake 'off' arm: SensAwake function off; OR

- SensAwake 'on' arm: SensAwake function on. When wakefulness is detected, SensAwake will automatically drop the pressure to the set SensAwake pressure. In the ICON+Auto Device, the minimum pressure is also the SensAwake pressure. The SensAwake pressure is the pressure that the device will drop to during wakefulness. So if the patient is experiencing a pressure of $12 \mathrm{~cm} \mathrm{H}_{2} \mathrm{O}$, and their SensAwake pressure is $4 \mathrm{~cm}$ $\mathrm{H}_{2} \mathrm{O}$, then it will drop from $12 \mathrm{~cm} \mathrm{H}_{2} \mathrm{O}$ to $4 \mathrm{~cm} \mathrm{H}_{2} \mathrm{O}$. The default SensAwake pressure is set to $4 \mathrm{~cm} \mathrm{H}_{2} \mathrm{O}$, however, for patients with higher therapeutic pressures, $4 \mathrm{~cm}$ may be too low and result in discomfort. Therefore, the SensAwake pressure can be increased to $6 \mathrm{~cm} \mathrm{H}_{2} \mathrm{O}$ to account for this.

The allocation list was computer generated by a statistician independent from the study investigators.

\section{Treatment}

Participants receive training in use of the AutoCPAP device as per usual care. Usual care is standardised across the participating centres. Participants receive in-home therapy for 4 weeks. During the last week of arm one, blood pressure is monitored over one 24-hour period, whereas the actimeter is worn during the 4 weeks of each treatment arm.

\section{Crossover}

Participants return to the centre for the crossover visit. Full AutoCPAP data (recorded by the device) and actigraphy data are downloaded. They again complete the questionnaires: ISI, ESS, PSQI and SF-12, and are issued with a new sleep diary. The patient's AutoCPAP device settings are switched over to the opposite treatment arm by the site coordinator without showing them to or discussing them with the patient or disclosing the settings to the physician. Participants receive at-home therapy for a further 4 weeks. During the last week of arm two, blood pressure is monitored over one 24-hour period.

\section{End of study}

Participants attend the study centre where full AutoCPAP data (entire folder on the device's USB) and actigraphy data are downloaded; they hand in their sleep diaries and again complete the ISI, ESS, PSQI and SF-12 questionnaires. If patients prefer the AutoCPAP with SensAwake, they will be able to continue to use this feature after the conclusion of the study. Participants may obtain a summary of trial results after these have been submitted for publication.

\section{Withdrawal and stopping criteria}

Patients have the right to withdraw from the study at any time. In addition, the investigators may withdraw a patient at any time for the following reasons: protocol violation, 
serious illness or adverse event. In the event of a serious adverse event, unblinding may be done through the site coordinator.

\section{Statistics}

\section{Sample size}

The sample size was calculated based on an assumption of a WASO of $58 \mathrm{~min} \pm \mathrm{SD}^{41}$ and allowing for the crossover nature of the study. It assumes that the $\mathrm{SD}$ of the difference between the two treatments is approximated by the SD derived from WASO single time assessments. The largest estimate of the WASO between individual SD was used: $46 \mathrm{~min}$ in the study by Natale $e t a l .{ }^{41}$ On this basis, a sample size of 40 completers of both treatments is required (2 sequence groups of 20/group each) to detect a difference of $15 \mathrm{~min}$ or more as statistically significant (two-tailed alpha $=0.05$ ) with $80 \%$ power. In a crossover study, it is advised to over-recruit to allow for dropouts, so the minimum sample size was set at 48 (24 per group) to allow for $20 \%$ dropout, with 12 patients per centre.

\section{Statistical analysis}

All consenting and enrolled patients will be included in the intention-to-treat analysis. Withdrawal and non-adherence to treatment are outcome measures, thus data on any withdrawn or non-adherent patients will be included.

A complete description of the study population will be presented with continuous variables expressed as median and IQR, and categorical variables as frequencies and percentages (see table 1 for all outcome measures by study visit or period).

Each 4-week treatment arm includes a first-week 'washout' period, where data will not be analysed.

Repeated measures analysis of variance (ANOVA) will be used to assess differences between the two treatments for actigraphy measures (WASO, TST, SOL, SE), treatment compliance, subjective questionnaire results (ISI, ESS, PSQI and SF-12) and 24 hours blood pressure measurements (minima, maxima, mean values of the systolic, diastolic and mean arterial pressures and dipping profile). If requirements for parametric repeated measures ANOVA are not met then a non-parametric Wilcoxon signed-rank test will be used. The analysis will include time-related factors (WASO, TST, SOL) and treatment as within-subject factors and treatment sequence as a between-subjects factor. The interaction between treatment and treatment sequence will be tested to ensure there are no carry-over effects influencing the comparison of the treatments. In the case of missing data for the primary outcome, it will be derived from the available WASO data at both post-treatment times (V2, V3). For both post-treatment times, missing data will be imputed from baseline outcome measures and any postbaseline assessments. Statistical data analysis will be performed using IBM SPSS Statistics V.22 and tested with a significance level of 0.05 , by an independent statistician.

Table 1 Outcome measurements at study visits

\begin{tabular}{|c|c|c|}
\hline Variable & Measurement & Measurement points* \\
\hline \multicolumn{3}{|l|}{ Primary end point } \\
\hline $\begin{array}{l}\text { Objective sleep quality } \\
\text { - Wake after sleep onset (WASO) }\end{array}$ & Actigraphy & $\begin{array}{l}\text { Days 1-7 (baseline) (downloaded at V1) } \\
\text { During week } 4 \text { of each treatment arm (days 29-35 and } \\
\text { days 56-63; downloaded at V2 and V3, respectively) }\end{array}$ \\
\hline \multicolumn{3}{|l|}{ Secondary end points } \\
\hline Treatment compliance & $\begin{array}{l}\text { CPAP (data recorded by } \\
\text { AutoCPAP) downloads }\end{array}$ & $\begin{array}{l}\text { Weeks } 2-4 \text { of each treatment arm (days } 15-35 \text { and days } \\
28-35 \text {; downloaded at V2 and V3, respectively) }\end{array}$ \\
\hline $\begin{array}{l}\text { Subjective sleep quality, insomnia, } \\
\text { daytime sleepiness and quality of life: } \\
\text { - Sleep quality } \\
\text { - Insomnia severity } \\
\text { Daytime sleepiness } \\
\text { - Quality of life }\end{array}$ & $\begin{array}{l}\text { Self-reporting } \\
\text { questionnaires: } \\
\text { PSQI, ISI, ESS, SF-12 }\end{array}$ & $\begin{array}{l}\text { Baseline (V1) on day } 7 \\
\text { Crossover visit (V2) on day } 35 \\
\text { End of study (V3) on day } 63\end{array}$ \\
\hline 24 hours blood pressure & $\begin{array}{l}24 \text { hours ambulatory } \\
\text { blood pressure monitor }\end{array}$ & $\begin{array}{l}24 \text { hours during days } 1-7 \text { (baseline) } \\
24 \text { hours during week } 4 \text { of treatment arm } 1 \text { (between day } \\
29 \text { and day } 35 \text { ) } \\
24 \text { hours during week } 4 \text { Treatment arm } 2 \text { (between day } 56 \\
\text { and day } 63 \text { ) }\end{array}$ \\
\hline
\end{tabular}

${ }^{*} A l l \pm 2$ days.

PSQI, Pittsburgh Sleep Quality Index; ISI, Insomnia Severity Index; ESS, Epworth Sleepiness Scale; SF-12, Short Form 12 Health Survey;V, study visit. 


\section{Ethics}

This study is conducted in accordance with the Declaration of Helsinki (last amended 2013), and the recommendations for Good Clinical Practice (GCP-ICHE6). The protocol (F\&P CIA-151 version $\mathrm{C}$ ) was approved by the French regional ethics committee (CPP Sud Est V, IRB $\mathrm{N}^{\circ} 6705$ ) on 9 December 2015. The final version (F\&P CIA-151 version E/ID-RCB: 2015-A00135-44) including changes requested by the French regulatory authority (ANSM) in February 2016 and amendments poststart of patient inclusions aimed at improving enrolment (inclusion period increased, Hospital Anxiety and Depression score $>11$ instead of 8) was approved on 2 May 2017. All patients are required to give written informed consent before inclusion in the study.

\section{Sponsor and coordination}

The study sponsor is Fisher \& Paykel Healthcare and was involved in study design. The sponsor holds insurance through Chubb Insurance, New Zealand Limited. The Grenoble-Alps University Hospital centre is coordinating the study and the principal investigator is Professor JeanLouis Pépin. Monitoring, vigilance and communication of important protocol modifications are delegated to the 'Direction de la Recherche Clinique' (Clinical Research Administration) at Grenoble Alps University Hospital, France.

\section{Confidentiality}

In accordance with French legislation, people with direct access to source data will take all necessary precautions to ensure the confidentiality of information relating to experimental, research and people who participate, in particular with regard to their identity and the results obtained.

The collected data will be made anonymous before being communicated to the sponsor (or to any other specified specialist). In no case will the names or addresses of the persons concerned appear. Anonymised data from the trial can be accessed by research members at the coordinating site only.

\section{DISCUSSION}

Both insomnia and OSA are frequent chronic diseases with numerous comorbidities and high health resource-related costs. A high degree of heterogeneity exists within patients with OSA regarding clinical presentation, risk factors and consequences. ${ }^{42}$ Interventional studies need to be conducted in specific subgroups of patients to progressively delineate personalised medicine for sleep apnoea. Likewise, insomnia has a broad spectrum of causes, among them being CPAP use. This is why we chose to include only those patients who are naïve to CPAP therapy, although this may restrict our enrolment rate.

Comorbid insomnia is often undiagnosed, undertreated or untreated in patients with OSA. Pharmacotherapy of insomnia is not recommended as a long-term treatment, ${ }^{43}$ but insomnia can reduce tolerance and adherence of patients to CPAP therapy., The addition of SensAwake to AutoCPAP may improve the comfort of AutoCPAP therapy, and therefore may increase sleep quality and duration, improve a patient's adherence to CPAP therapy and improve quality of life. One strength of our study is to combine objective assessments of sleep indices and patient-centred outcomes. As the addition of insomnia to OSA increases the prevalence of hypertension, ${ }^{44}$ the valuation of blood pressure by means of 24 hours ambulatory blood pressure monitoring adds another strength to the study. We will also investigate whether conjointly improving sleep apnoea and sleep duration/quality will allow a better control of nocturnal blood pressure.

The results of this study will help both OSA and insomnia specialists in their decision as whether to prescribe AutoCPAP with or without SensAwake for CPAP treatment of patients with OSA who also have insomnia. If positive, this study will be a step forward for personalised therapy in the frequent subgroup of OSA plus insomnia.

\section{Author affiliations}

${ }^{1}$ Hypoxia-pathophysiology Laboratory - INSERM U1042, Grenoble Alps University Hospital, Université Grenoble Alpes, Grenoble, France

2Department of Pneumology, INSERM URM 1063, Angers University Hospital,

Bretagne Loire University I, Angers, France

${ }^{3}$ Fisher \& Paykel Healthcare Limited, Auckland, New Zealand

${ }^{4}$ Sleep Clinic - Lariboisière Hospital, Assistance Publique-Hopitaux de Paris, Paris, Île-de-France, France

${ }^{5}$ Department of Physiology and Functional Exploration, Bichat Hospital, Denis Diderot University, Paris, France

Acknowledgements The authors would like to thank Drs Marie Destors, Pascaline Priou, Wojtek Trzepizur, Helene Benzaquen and Ruben Wanono for their advice and participation, Marie Peeters for trial management and Rebecca Thomson for help in revising the manuscript.

Contributors JLP, FG, RV, BO, MPO, WVB and RT designed the study and wrote the study protocol. AF and JLP wrote the article based on the study protocol. FG, MPO, $\mathrm{RV}$ and $\mathrm{BO}$ critically revised the manuscript. MB calculated the sample size and wrote the study statistical analysis plan. JLP, FG, MPO, VBB and RT are currently including patients in the study. All authors approved the submitted manuscript.

Funding This study is funded by unrestricted grant from Fisher \& Paykel Healthcare Limited (Auckland, New Zealand).

Competing interests RV and BO are employed by Fisher \& Paykel Healthcare Limited.

Patient consent Study protocol: the inclusion of patients is ongoing. Written informed consent is an inclusion criterion.

Ethics approval French CPP Sud-Est V, IRB Nº6705.

Provenance and peer review Not commissioned; externally peer reviewed.

Open Access This is an Open Access article distributed in accordance with the Creative Commons Attribution Non Commercial (CC BY-NC 4.0) license, which permits others to distribute, remix, adapt, build upon this work non-commercially, and license their derivative works on different terms, provided the original work is properly cited and the use is non-commercial. See: http://creativecommons.org/ licenses/by-nc/4.0/

C Article author(s) (or their employer(s) unless otherwise stated in the text of the article) 2017. All rights reserved. No commercial use is permitted unless otherwise expressly granted. 


\section{REFERENCES}

1. Lévy P, Kohler M, McNicholas WT, et al. Obstructive sleep apnoea syndrome. Nat Rev Dis Primers 2015;1:15015.

2. Peppard PE, Young T, Barnet JH, et al. Increased prevalence of sleep-disordered breathing in adults. Am J Epidemiol 2013;177:1006-14.

3. Sullivan CE, Issa FG, Berthon-Jones M, et al. Reversal of obstructive sleep apnoea by continuous positive airway pressure applied through the nares. Lancet 1981;1:862-5.

4. Gay P, Weaver T, Loube D, et al. Evaluation of positive airway pressure treatment for sleep related breathing disorders in adults. Sleep 2006;29:381-401.

5. Weaver TE, Kribbs NB, Pack Al, et al. Night-to-night variability in CPAP use over the first three months of treatment. Sleep 1997;20:278-83.

6. Kribbs NB, Pack Al, Kline LR, et al. Objective measurement of patterns of nasal CPAP use by patients with obstructive sleep apnea. Am Rev Respir Dis 1993;147:887-95.

7. Luyster FS, Buysse DJ, Strollo PJ. Comorbid insomnia and obstructive sleep apnea: challenges for clinical practice and research. J Clin Sleep Med 2010;6:196-204.

8. Berry RB, Parish JM, Hartse KM. The use of auto-titrating continuous positive airway pressure for treatment of adult obstructive sleep apnea. Sleep 2002;25:148-73.

9. Oksenberg A, Silverberg DS, Arons E, et al. The sleep supine position has a major effect on optimal nasal continuous positive airway pressure : relationship with rapid eye movements and non-rapid eye movements sleep, body mass index, respiratory disturbance index, and age. Chest 1999;116:1000-6.

10. Ayas NT, Patel SR, Malhotra A, et al. Auto-titrating versus standard continuous positive airway pressure for the treatment of obstructive sleep apnea: results of a meta-analysis. Sleep 2004;27:249-53.

11. Behbehani K, Yen FC, Lucas EA, et al. A sleep laboratory evaluation of an automatic positive airway pressure system for treatment of obstructive sleep apnea. Sleep 1998;21:485-91.

12. Boudewyns A, Grillier-Lanoir V, Willemen MJ, et al. Two months follow up of auto-CPAP treatment in patients with obstructive sleep apnoea. Thorax 1999;54:147-9.

13. d'Ortho MP. Auto-titrating continuous positive airway pressure for treating adult patients with sleep apnea syndrome. Curr Opin Pulm Med 2004;10:495-9.

14. d'Ortho MP, Grillier-Lanoir V, Levy P, et al. Constant vs. automatic continuous positive airway pressure therapy: home evaluation. Chest 2000;118:1010-7.

15. Randerath WJ, Schraeder O, Galetke W, et al. Autoadjusting CPAP therapy based on impedance efficacy, compliance and acceptance. Am J Respir Crit Care Med 2001;163:652-7.

16. Teschler H, Berthon-Jones M, Thompson AB, et al. Automated continuous positive airway pressure titration for obstructive sleep apnea syndrome. Am J Respir Crit Care Med 1996;154:734-40.

17. Hudgel DW, Fung C. A long-term randomized, cross-over comparison of auto-titrating and standard nasal continuous airway pressure. Sleep 2000;23:1-4.

18. Hukins C. Comparative study of autotitrating and fixed-pressure CPAP in the home: a randomized, single-blind crossover trial. Sleep 2004;27:1512-7.

19. Konermann M, Sanner BM, Vyleta M, et al. Use of conventional and self-adjusting nasal continuous positive airway pressure for treatment of severe obstructive sleep apnea syndrome: a comparative study. Chest 1998;113:714-8.

20. Meurice JC, Marc I, Sériès F. Efficacy of auto-CPAP in the treatment of obstructive sleep apnea/hypopnea syndrome. Am J Respir Crit Care Med 1996;153:794-8.

21. Randerath WJ, Galetke W, David M, et al. Prospective randomized comparison of impedance-controlled auto-continuous positive airway pressure (APAP(FOT)) with constant CPAP. Sleep Med $2001 ; 2: 115-24$.
22. Teschler H, Wessendorf TE, Farhat AA, et al. Two months autoadjusting versus conventional nCPAP for obstructive sleep apnoea syndrome. Eur Respir J 2000;15:990-5.

23. To KW, Chan WC, Choo KL, et al. A randomized cross-over study of auto-continuous positive airway pressure versus fixed-continuous positive airway pressure in patients with obstructive sleep apnoea. Respirology 2008;13:79-86.

24. Ayappa I, Norman RG, Whiting D, et al. Irregular respiration as a marker of wakefulness during titration of CPAP. Sleep 2009;32:99-104.

25. Dungan GC, Marshall NS, Hoyos CM, et al. A randomized crossover trial of the effect of a novel method of pressure control (SensAwake) in automatic continuous positive airway pressure therapy to treat sleep disordered breathing. J Clin Sleep Med 2011;7:261-7.

26. Cumin D, Whiting D, Malla A, et al. T-I-065 randomized crossover evaluation of a novel implementation of pressure relief technology sensawake $^{\mathrm{TM}}$ and fixed pressure cpap. Sleep Med 2011;12:S75.

27. Powell ED, Overstreet DR, Uhles ML, et al. T-I-054 Interim analysis of long-term patient feedback on a novel automatic ramp feature in fixed pressure cpap. Sleep Med 2011;12:S72.

28. Glidewell RN, Renn BN, Roby E, et al. Predictors and patterns of insomnia symptoms in OSA before and after PAP therapy. Sleep Med 2014;15:899-905.

29. Bjorvatn B, Pallesen S, Grønli J, et al. Prevalence and correlates of insomnia and excessive sleepiness in adults with obstructive sleep apnea symptoms. Percept Mot Skills 2014;118:571-86.

30. Mysliwiec V, Matsangas P, Baxter T, et al. Comorbid insomnia and obstructive sleep apnea in military personnel: correlation with polysomnographic variables. Mil Med 2014;179:294-300.

31. Björnsdóttir E, Janson C, Sigurdsson JF, et al. Symptoms of insomnia among patients with obstructive sleep apnea before and after two years of positive airway pressure treatment. Sleep 2013;36:1901-9.

32. Lichstein $\mathrm{KL}$, Justin Thomas S, Woosley JA, et al. Co-occurring insomnia and obstructive sleep apnea. Sleep Med 2013;14:824-9.

33. Wickwire EM, Smith MT, Birnbaum S, et al. Sleep maintenance insomnia complaints predict poor CPAP adherence: A clinical case series. Sleep Med 2010;11:772-6.

34. Pieh C, Bach M, Popp R, et al. Insomnia symptoms influence CPAP compliance. Sleep Breath 2013;17:99-104.

35. Gagnon $\mathrm{C}$, Bélanger L, Ivers $\mathrm{H}$, et al. Validation of the insomnia severity index in primary care. J Am Board Fam Med 2013;26:701-10.

36. wGT3X-BT. ActiGraph wGT3X-BT. http://actigraphcorp.com/ products-showcase/activity-monitors/actigraph-wgt3x-bt/ (accessed Jul 2017).

37. Cole RJ, Kripke DF, Gruen W, et al. Automatic sleep/wake indentification from wrist activity. Sleep 1992;12:461-9.

38. Kaminska M, Jobin V, Mayer P, et al. The epworth sleepiness scale: self-administration versus administration by the physician, and validation of a French version. Can Respir J 2010;17:e27-e34.

39. Blais FC, Gendron L, Mimeault V, et al. [Evaluation of insomnia: validity of 3 questionnaires]. Encephale 1997;23:447-53.

40. Gandek B, Ware JE, Aaronson NK, et al. Cross-validation of item selection and scoring for the SF-12 Health Survey in nine countries: results from the IQOLA Project. International Quality of Life Assessment. J Clin Epidemiol 1998;51:1171-8.

41. Natale V, Léger D, Martoni M, et al. The role of actigraphy in the assessment of primary insomnia: a retrospective study. Sleep Med 2014;15:111-5.

42. Bailly S, Destors M, Grillet Y, et al. Obstructive sleep apnea: a cluster analysis at time of diagnosis. PLoS One 2016;11:e0157318.

43. Lack L, Sweetman A. Diagnosis and treatment of insomnia comorbid with obstructive sleep apnea. Sleep Med Clin 2016;11:379-88.

44. Gupta MA, Knapp K. Cardiovascular and psychiatric morbidity in obstructive sleep apnea (OSA) with insomnia (sleep apnea plus) versus obstructive sleep apnea without insomnia: a case-control study from a Nationally Representative US sample. PLoS One 2014;9:e90021. 